\title{
Derivation method of numerous dynamics in the Special Theory of Relativity
}

https://doi.org/10.1515/phys-2019-0016

Received May 19, 2018; accepted August 10, 2018

\begin{abstract}
The article presents innovative method of deriving dynamics in the Special Theory of Relativity. This method enables to derive infinitely many dynamics in relativistic mechanics. The authors have shown five examples of these derivations. In this way, It is presented that the dynamics known today as the dynamics of Special Theory of Relativity is only one of infinitely of theoretically possible.
\end{abstract}

Keywords: dynamics of bodies, Special Theory of Relativity

PACS: 03.30. $+\mathrm{p}, 02.90 .+\mathrm{p}$

\section{Introduction}

The currently accepted dynamics of STR have different experimental confirmations. Present article does not deal with these experiments. They are not analyzed, nor evaluated. The purpose of this article is to show that the concrete dynamics of STR does not only result from the kinematics of STR only. Formally and correctly mathematically, many STR dynamics, which have different properties, can be derived within the kinematics STR. Relativistic dynamics is derived based on the relativistic kinematics and one additional assumption, which allows the concept of mass, momentum and kinetic energy to be introduced into the theory. This paper is a discussion about possible assumptions and the dynamics of STR resulting from these assumptions. It presents the author's method of deriving numerous dynamics for this theory.

The decision, in which dynamic STR is correct, can only result from experiments. Available publications show that the dynamics indicated by Albert Einstein is correct, that is for $x=3 / 2$ (see Section 5). However, because each experiment is fraught with errors, it is possible that more

*Corresponding Author: Roman Szostek: Rzeszow University of Technology, Department of Quantitative Methods, Rzeszow, Poland, E-mail: rszostek@prz.edu.pl accurate experiments carried out in the future will show that the optimal model of dynamics for $x=3 / 2 \pm \Delta x$, where $\Delta x$ is a noticeable correction.

Kinematics deals with the movement of bodies without taking their physical characteristics into account. The basic concepts of kinematics are: time, location, transformation, speed and acceleration.

Dynamics deals with the movement of material bodies under the action of forces. The basic concepts of dynamics are: inertial mass, force, momentum and kinetic energy.

Kinematics and dynamics are resulting in mechanics. This study deals with relativistic mechanics, i.e. the Special Theory of Relativity, which unlike classical mechanics, also applies to high-speed.

\section{Kinematic assumptions of the Special Theory of Relativity}

The kinematics of the Special Theory of Relativity is based on the following assumptions:

\section{All inertial systems are equivalent.}

This assumption means that there is no physical phenomenon, which distinguishes the inertial system. In a particular case, it means that there is no such phenomenon for which the absolute rest is needed to explain. Mathematically, it results from this assumption that each coordinate and time transformation have coefficients with exactly the same numerical values as inverse transformation (with the accuracy to the sign resulting from the velocity direction between the systems).

II. Velocity of light $c$ in vacuum is the same in every direction and in each inertial system.

III. Transformation of time and position coordinates between the inertial systems is linear.

These assumptions are often written in other equivalent forms.

Based on mentioned assumptions, it is possible to derive Lorentz transformation on which the Special Theory of Relativity is based. There are many different derivation 
ways of this transformation. Two derivations are presented in monograph [1].

Markings adopted in Figure 1 will be convenient for our needs. Inertial systems move along their $x$-axis. Symbol $v_{2 / 1}$ stands for a velocity of $U_{2}$ system measured by the observer from $U_{1}$ system, while $v_{1 / 2}$ is a velocity of $U_{1}$ system measured by the observer from $U_{2}$ system. In the Special Theory of Relativity occurs that $v_{2 / 1}=-v_{1 / 2}$.

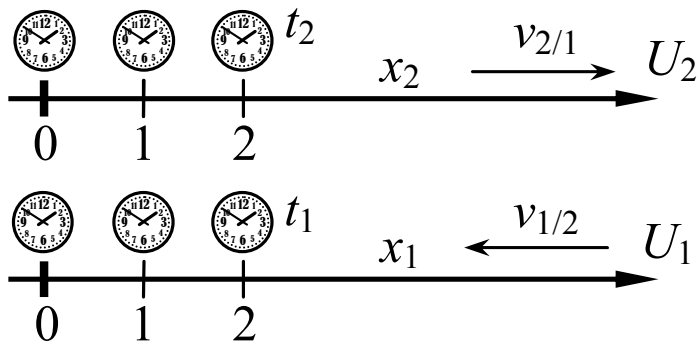

Figure 1: Relative movement of inertial systems $U_{1}$ and $U_{2}$ $\left(\mathrm{v}_{2 / 1}=-\mathrm{v}_{1 / 2}\right)$

Lorentz transformation from $U_{2}$ to $U_{1}$ system has a form of:

$$
\begin{gathered}
t_{1}=\frac{1}{\sqrt{1-\left(v_{2 / 1} / c\right)^{2}}}\left(t_{2}+\frac{v_{2 / 1}}{c^{2}} x_{2}\right) \\
x_{1}=\frac{1}{\sqrt{1-\left(v_{2 / 1} / c\right)^{2}}}\left(v_{2 / 1} t_{2}+x_{2}\right) \\
y_{1}=y_{2}, \quad z_{1}=z_{2}
\end{gathered}
$$

Lorentz transformation from $U_{1}$ to $U_{2}$ system has a form of:

$$
\begin{gathered}
t_{2}=\frac{1}{\sqrt{1-\left(v_{1 / 2} / c\right)^{2}}}\left(t_{1}+\frac{v_{1 / 2}}{c^{2}} x_{1}\right) \\
x_{2}=\frac{1}{\sqrt{1-\left(v_{1 / 2} / c\right)^{2}}}\left(v_{1 / 2} t_{1}+x_{1}\right) \\
y_{2}=y_{1}, \quad z_{2}=z_{1}
\end{gathered}
$$

Transformation (1) - (3) and (4) - (6) includes complete information on the relativistic kinematics.

\section{Selected properties of relativistic kinematics}

In order to derive dynamics two formulas from kinematics, i.e. (20) and (23) from kinematics will be needed. They will be derived out of transformation (1) - (3).

\subsection{Transformation of velocity}

Determine the differentials from transformation (1) - (3)

$$
\begin{gathered}
d t_{1}=\frac{1}{\sqrt{1-\left(v_{2 / 1} / c\right)^{2}}}\left(d t_{2}+\frac{v_{2 / 1}}{c^{2}} d x_{2}\right) \\
d x_{1}=\frac{1}{\sqrt{1-\left(v_{2 / 1} / c\right)^{2}}}\left(v_{2 / 1} d t_{2}+d x_{2}\right) \\
d y_{1}=d y_{2}, \quad d z_{1}=d z_{2}
\end{gathered}
$$

From the inertial system $U_{1}$ and $U_{2}$, the moving body $U_{3}$ is observed. In $U_{1}$ system, it has a velocity of $v_{3 / 1}$, while in $U_{2}$ system it has a velocity of $v_{3 / 2}$. The components of these velocities are presented in Figure 2.
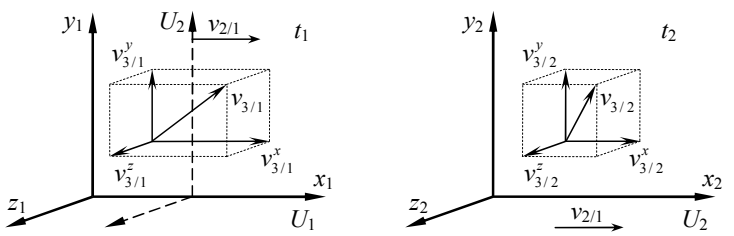

Figure 2: Movement of the body from two inertial systems $U_{1}$ and $U_{2}$

The coordinates of body $U_{3}$ position in $U_{1}$ system are $x_{1}, y_{1}, z_{1}$. At the same time in $U_{2}$ system these coordinates are $x_{2}, y_{2}, z_{2}$. Since the body $U_{3}$ moves, these coordinates change in time. When time $d t_{1}$ elapses in $U_{1}$ system then time $d t_{2}$ elapses in $U_{2}$ system. For such indications the changes of coordinates of body $U_{3}$ position in $U_{1}$ system in the time interval $d t_{1}$ are $d x_{1}, d y_{1}, d z_{1}$. Changes of coordinates of body $U_{3}$ position in $U_{2}$ system in the time interval $d t_{2}$ are $d x_{2}, d y_{2}, d z_{2}$.

The body velocity $U_{3}$ in inertial system $U_{2}$ has the following components:

$$
v_{3 / 2}^{x}=\frac{d x_{2}}{d t_{2}}, \quad v_{3 / 2}^{y}=\frac{d y_{2}}{d t_{2}}, \quad v_{3 / 2}^{z}=\frac{d z_{2}}{d t_{2}}
$$

The body velocity $U_{3}$ in inertial system $U_{1}$ has the following components:

$$
v_{3 / 1}^{x}=\frac{d x_{1}}{d t_{1}}, \quad v_{3 / 1}^{y}=\frac{d y_{1}}{d t_{1}}, \quad v_{3 / 1}^{z}=\frac{d z_{1}}{d t_{1}}
$$


When differentials (7) - (9) are put into Eqs. (11), one will receive

$$
\left\{\begin{array}{l}
v_{3 / 1}^{X}=\frac{\frac{1}{\sqrt{1-\left(v_{2 / 1} / c^{2}\right.}}\left(v_{2 / 1} d t_{2}+d x_{2}\right)}{\frac{1}{\sqrt{1-\left(v_{2 / 1} / c\right)^{2}}}\left(d t_{2}+\frac{v_{2 / 1}}{c^{2}} d x_{2}\right)} \\
v_{3 / 1}^{y}=\frac{1}{\frac{1}{\sqrt{1-\left(v_{2 / 1} / c\right)^{2}}}\left(d t_{2}+\frac{v_{2 / 1}}{c^{2}} d x_{2}\right)} \\
v_{3 / 1}^{z}=\frac{d z_{2}}{\frac{1}{\sqrt{1-\left(v_{2 / 1} / c\right)^{2}}}\left(d t_{2}+\frac{v_{2 / 1}}{c^{2}} d x_{2}\right)}
\end{array}\right.
$$

i.e.

$$
\left\{\begin{array}{l}
v_{3 / 1}^{x}=\frac{v_{2 / 1}+d x_{2} / d t_{2}}{1+\frac{v_{2 / 1}}{c^{2}}\left(d x_{2} / d t_{2}\right)} \\
v_{3 / 1}^{y}=\sqrt{1-\left(v_{2 / 1} / c\right)^{2}} \frac{d y_{2} / d t_{2}}{1+\frac{v_{2 / 1}}{c^{2}}\left(d x_{2} / d t_{2}\right)} \\
v_{3 / 1}^{z}=\sqrt{1-\left(v_{2 / 1} / c\right)^{2}} \frac{d z_{2} / d t_{2}}{1+\frac{v_{2 / 1}}{c^{2}}\left(d x_{2} / d t_{2}\right)}
\end{array}\right.
$$

On the basis of (10) the desired velocity transformation from $U_{2}$ to $U_{1}$ system is obtained

$$
\left\{\begin{array}{l}
v_{3 / 1}^{X}=\frac{v_{3 / 2}^{x}+v_{2 / 1}}{1+\frac{v_{3 / 2} v_{2 / 1}}{c^{2}}} \\
v_{3 / 1}^{y}=\sqrt{1-\left(v_{2 / 1} / c\right)^{2}} \frac{v_{3 / 2}^{y}}{1+\frac{v_{3 / 2}^{x} v_{2 / 1}}{c^{2}}} \\
v_{3 / 1}^{z}=\sqrt{1-\left(v_{2 / 1} / c\right)^{2}} \frac{v_{3 / 2}^{z}}{1+\frac{v_{3 / 2}^{z} v_{2 / 1}}{c^{2}}}
\end{array}\right.
$$

In special case, when $U_{3}$ body moves parallel to $x$-axis then occurs

$$
v_{3 / 1}^{x}=v_{3 / 1}, v_{3 / 2}^{x}=v_{3 / 2}, v_{3 / 1}^{y}=v_{3 / 2}^{y}=0, v_{3 / 1}^{z}=v_{3 / 2}^{z}=0
$$

Then velocity transformation (14) takes the form of formula to sum-up parallel velocities

$$
v_{3 / 1}=\frac{v_{3 / 2}+v_{2 / 1}}{1+\frac{v_{3 / 2} v_{2 / 1}}{c^{2}}}
$$

\subsection{Change of velocity seen from different inertial systems}

The body at rest in $U_{3}$ system has momentary acceleration to $U_{3^{\prime}}$ system. The body movement is observed from $U_{1}$ and $U_{2}$ systems. The velocities of inertial systems are parallel to each other. Markings shown in Figure 3 are adopted.

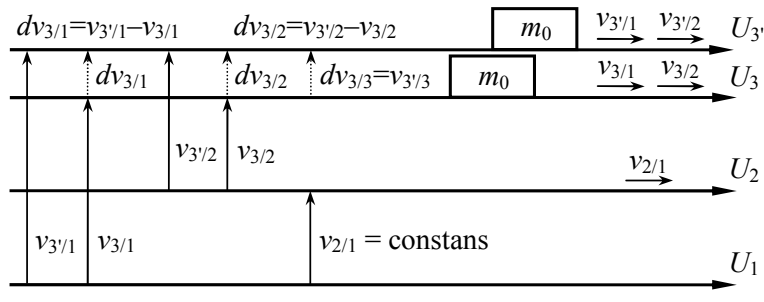

Figure 3: Increases in the velocity seen in inertial systems $U_{1}$ and $U_{2}$

The differentials from formula (16) will be determined:

$$
\begin{aligned}
d v_{3 / 1}= & \frac{d \frac{v_{3 / 2}+v_{2 / 1}}{1+\left(v_{3 / 2} v_{2 / 1} / c^{2}\right.}}{d v_{3 / 2}} d v_{3 / 2} \\
= & \frac{1+\frac{v_{3 / 2} v_{2 / 1}}{c^{2}}-\left(v_{3 / 2}+v_{2 / 1}\right) \frac{v_{2 / 1}}{c^{2}}}{\left(1+\frac{v_{3 / 2} v_{2 / 1}}{c^{2}}\right)^{2}} d v_{3 / 2} \\
& d v_{3 / 1}=\frac{1-\frac{v_{2 / 1}^{2}}{c^{2}}}{\left(1+\frac{v_{3 / 2} v_{2 / 1}}{c^{2}}\right)^{2}} d v_{3 / 2}
\end{aligned}
$$

If $U_{3}$ system is $U_{2}$ system then it is necessary to replace index 3 with 2 . Then,

$$
d v_{3 / 1}=d v_{2 / 1}, \quad v_{3 / 2}=v_{2 / 2}=0, \quad d v_{3 / 2}=d v_{2 / 2}
$$

On this basis, the formula (18) takes a form of

$$
d v_{2 / 2}=\frac{d v_{2 / 1}}{1-\left(v_{2 / 1} / c\right)^{2}}
$$

Relation (20) is related to the change of body velocity seen in the inertial system $U_{2}$, in which the body is located $\left(d v_{2 / 2}\right)$, and the change of velocity seen from another inertial system $U_{1}\left(d v_{2 / 1}\right)$.

\subsection{Time dilatation}

If motionless body is in $U_{2}$ system, then for its coordinates occurs

$$
\frac{d x_{2}}{d t_{2}}=0
$$

Based on time transformation (7) one receives

$$
\begin{aligned}
& \frac{d t_{1}}{d t_{2}}=\frac{1}{\sqrt{1-\left(v_{2 / 1} / c\right)^{2}}}\left(1+\frac{v_{2 / 1}}{c^{2}} \frac{d x_{2}}{d t_{2}}\right) \stackrel{\frac{d x_{2}}{d d 2_{2}}=0}{\Rightarrow} \\
& \frac{d t_{1}}{d t_{2}}=\frac{1}{\sqrt{1-\left(v_{2 / 1} / c\right)^{2}}}
\end{aligned}
$$

On this basis we receive the formula for time dilatation of motionless body with regard to $U_{2}$ system

$$
\frac{d x_{2}}{d t_{2}}=0 \Rightarrow d t_{2}=\sqrt{1-\left(v_{2 / 1} / c\right)^{2}} \cdot d t_{1}
$$


Recording of time dilatation in a form of (23) is more precise than the commonly used recording, as it has a form of implications. Such a record makes it clear that such dilatation applies only to motionless bodies in relation to $U_{2}$ system (or for events occurring in the same position in relation to $U_{2}$ system).

\section{Dynamics in the Special Theory of Relativity}

All dissertations will be conducted only for onedimensional model, i.e. all analyzed vector values will be parallel to $x$-axis. Each derived dynamic can easily be generalized into three-dimensional cases.

In order to derive dynamics in the Special Theory of Relativity (STR), it is necessary to adopt an additional assumption, which allows the concept of mass, momentum and kinetic energy to be introduced into the theory. Depending on the assumption, different dynamics of bodies are received.

The inertial mass body resting in inertial frame of reference is determined by $m_{0}$ (rest mass). The rest mass is determined on the base unit of mass and the method of comparing any mass with this base unit. The inertial mass body at rest in $U_{2}$, as seen from $U_{1}$ system, is determined by $m_{2 / 1}$ (relativistic mass). It is worth to note that the relativistic mass in this case is an inertial mass that occurs in the Newton's second law, rather than mass occurring in the formula for momentum, as assumed in the STR. In this way, a different definition of relativistic mass has been adopted, than one in the STR. Such a definition of the relativistic mass is more convenient in deriving dynamics.

The body of $m_{0}$ inertial mass is in $U_{2}$ system. It is affected by force $F_{2 / 2}$ that causes acceleration of $d v_{2 / 2} / d t_{2}$. Therefore, for the observer from $U_{2}$ system, the Newton's second law takes a form of

$$
F_{2 / 2}:=m_{0} \cdot a_{2 / 2}=m_{0} \frac{d v_{2 / 2}}{d t_{2}}
$$

For the observer from $U_{1}$ system, inertial mass of the same body is $m_{2 / 1}$. For this observer, the force $F_{2 / 1}$ acts on the body, causing acceleration of $d v_{2 / 1} / d t_{1}$. Therefore, for the observer from $U_{1}$ the Newton's second law takes the form of

$$
\begin{aligned}
F_{2 / 1} & :=f\left(v_{2 / 1}\right) \cdot m_{0} \cdot a_{2 / 1}=m_{2 / 1}\left(v_{2 / 1}\right) \cdot a_{2 / 1} \\
& =m_{2 / 1} \cdot a_{2 / 1}=m_{2 / 1} \frac{d v_{2 / 1}}{d t_{1}}
\end{aligned}
$$

Equation (25) means that a generalized form of the Newton's second law is postulated. This generalized form contains an additional parameter $f(v)$. From the formula (24) shows that always $f(0)=1$. In classical mechanics $f(v)=1$, while in the current dynamics STR $f(v)=\gamma^{3}$ (formula (32)). Determining another form of parameter $f(v)$ leads to other dynamics for STR. The inertial relativistic mass $m_{2 / 1}$ is the product of this additional parameter $f(v)$ and the inertial mass body at rest $m_{0}$. In this article, the parameter $f(v)$ will not be used, only the inertial relativistic mass $m_{2 / 1}$.

Definitions identical as in classical mechanics apply for momentum and kinetic energy.

For the observer from $U_{2}$ system, the change of this body momentum can be recorded in the following forms

$$
\begin{aligned}
d p_{2 / 2} & :=F_{2 / 2} \cdot d t_{2}=m_{0} \cdot a_{2 / 2} \cdot d t_{2}=m_{0} \frac{d v_{2 / 2}}{d t_{2}} d t_{2} \\
& =m_{0} \cdot d v_{2 / 2}
\end{aligned}
$$

For the observer from $U_{1}$ system, the change of this body momentum can be recorded in the following forms

$$
\begin{aligned}
d p_{2 / 1} & :=F_{2 / 1} \cdot d t_{1}=m_{2 / 1} \cdot a_{2 / 1} \cdot d t_{1}=m_{2 / 1} \frac{d v_{2 / 1}}{d t_{1}} d t_{1} \\
& =m_{2 / 1} \cdot d v_{2 / 1}
\end{aligned}
$$

where:

$-d p_{2 / 2}$ is a change of body momentum with rest mass $m_{0}$ in the inertial system $U_{2}$, measured by the observer from the same inertial system $U_{2}$,

$-d p_{2 / 1}$ is a change of body momentum in the inertial system $U_{2}$, measured by the observer from the same inertial system $U_{1}$.

Kinetic energy of the body is equal of the work into its acceleration. For the observer from $U_{1}$ system, the change of kinetic energy of this body is as follows

$$
\begin{aligned}
d E_{2 / 1} & :=F_{2 / 1} \cdot d x_{2 / 1}=m_{2 / 1} \cdot a_{2 / 1} \cdot d x_{2 / 1} \\
& =m_{2 / 1} \frac{d v_{2 / 1}}{d t_{1}} d x_{2 / 1}=m_{2 / 1} \frac{d x_{2 / 1}}{d t_{1}} d v_{2 / 1} \\
& =m_{2 / 1} \cdot v_{2 / 1} \cdot d v_{2 / 1}
\end{aligned}
$$

where:

$-d E_{2 / 1}$ is a change of kinetic energy of the body in inertial system $U_{2}$, measured by the observer from the inertial system $U_{1}$.

\subsection{STR dynamics with constant force (STR/F)}

In this section, a model of dynamics of bodies based on the assumption that the force accelerating of the body (parallel to $x$-axis) is the same for an observer from every inertial system will be derived (hence indication $F$ ). 


\subsubsection{The relativistic mass in STR/F}

In the model STR $/ F$ it is assumed, that

$$
F_{2 / 1}^{F}:=F_{2 / 2}
$$

Having introduced (24) and (25), one obtains

$$
m_{2 / 1}^{F} \frac{d v_{2 / 1}}{d t_{1}}=m_{0} \frac{d v_{2 / 2}}{d t_{2}}
$$

On the base (20) and (23), one has

$$
m_{2 / 1}^{F} \frac{d v_{2 / 1}}{d t_{1}}=m_{0} \frac{\frac{d v_{2 / 1}}{1-\left(v_{2 / 1} / c\right)^{2}}}{\sqrt{1-\left(v_{2 / 1} / c\right)^{2}} \cdot d t_{1}}
$$

Hence, a formula for relativistic mass of the body that is located in the system $U_{2}$ and is seen from the system $U_{1}$ is obtained, when assumption (29) is satisfied, as below

$$
m_{2 / 1}^{F}=m_{0}\left[\frac{1}{1-\left(v_{2 / 1} / c\right)^{2}}\right]^{3 / 2}
$$

\subsubsection{The momentum in STR/F}

The body of rest mass $m_{0}$ is associated with the system $U_{2}$. To determine the momentum of the body relative to the system $U_{1}$ a substitution of (32) to (27)

$$
\begin{aligned}
d p_{2 / 1}^{F} & =m_{2 / 1}^{F} \cdot d v_{2 / 1}=m_{0}\left[\frac{1}{1-\left(v_{2 / 1} / c\right)^{2}}\right]^{3 / 2} d v_{2 / 1} \\
& =m_{0} c^{3} \frac{1}{\left(c^{2}-v_{2 / 1}^{2}\right)^{3 / 2}} d v_{2 / 1}
\end{aligned}
$$

The body momentum is a sum of increases in its momentum, when the body is accelerated from the inertial system $U_{1}$ (the body has velocity 0 ) to the inertial system $U_{2}$ (the body has velocity $v_{2 / 1}$ ), i.e.

$$
p_{2 / 1}^{F}=m_{0} c^{3} \int_{0}^{v_{2 / 1}} \frac{1}{\left(c^{2}-v_{2 / 1}^{2}\right)^{3 / 2}} d v_{2 / 1}
$$

From the work [2] (formula 72, p. 167) it is possible to read out, that

$$
\int \frac{d x}{\left(a^{2}-x^{2}\right)^{3 / 2}}=\frac{x}{a^{2} \sqrt{a^{2}-x^{2}}}, \quad a \neq 0
$$

After applying the integral (35) to (34) the formula for the body momentum in $U_{2}$ system is received and measured by the observer from $U_{1}$ system in a form of

$$
p_{2 / 1}^{F}=m_{0} c^{3} \frac{v_{2 / 1}}{c^{2} \sqrt{c^{2}-v_{2 / 1}^{2}}}=\frac{m_{0}}{\sqrt{1-\left(v_{2 / 1} / c\right)^{2}}} v_{2 / 1}
$$

This formula is identical to the formula for momentum known from the STR, for the same reasons as in the case of momentum. This is because the dynamics known from the STR is derived from the assumption (29). It was adopted unconsciously, because it was considered as necessary. The awareness of this assumption allows to its change and derives other dynamics.

As already mentioned above, the definition of relativistic mass adopted is different from the definition adopted in the STR. In this case, the relativistic mass is the one, which occurs in the Newton's second law (25). In this particular case, it is expressed in terms of dependency (32). In the STR, the relativistic mass is the one, which occurs in the formula (36) per momentum.

\subsubsection{The momentum in STR/F for small velocities}

For small velocity $v_{2 / 1}<<c$ momentum (36) comes down to the momentum from classical mechanics, because

$$
v_{2 / 1} \ll c \Rightarrow p_{2 / 1}^{F} \approx m_{0} v_{2 / 1}
$$

\subsubsection{The kinetic energy in STR/F}

A determination of the formula for kinetic energy will be given. The dependence for the relativistic mass (32) is introduced to the formula (28)

$$
\begin{aligned}
d E_{2 / 1}^{F} & =m_{2 / 1}^{F} \cdot v_{2 / 1} \cdot d v_{2 / 1}=m_{0}\left[\frac{1}{1-\left(v_{2 / 1} / c\right)^{2}}\right]^{3 / 2} v_{2 / 1} d v_{2 / 1} \\
& =m_{0} c^{3} \frac{v_{2 / 1}}{\left(c^{2}-v_{2 / 1}^{2}\right)^{3 / 2}} d v_{2 / 1}
\end{aligned}
$$

The kinetic energy of body is a sum of increases in its kinetic energy, when the body is accelerated from the inertial system $U_{1}$ (the body has velocity 0 ) to the inertial system $U_{2}$ (the body has velocity $v_{2 / 1}$ ), i.e.

$$
E_{2 / 1}^{F}=m_{0} c^{3} \int_{0}^{v_{2 / 1}} \frac{v_{2 / 1}}{\left(c^{2}-v_{2 / 1}^{2}\right)^{3 / 2}} d v_{2 / 1}
$$

From the work [2] (formula 74, p. 167) it is possible to read out, that

$$
\int \frac{x d x}{\left(a^{2}-x^{2}\right)^{3 / 2}}=\frac{1}{\sqrt{a^{2}-x^{2}}}
$$

After applying the integral (40) to (39) the formula for the kinetic energy of the body in $U_{2}$ system and measured 
by the observer from $U_{1}$ system in a form of

$$
\begin{aligned}
E_{2 / 1}^{F} & =\left.m_{0} c^{3} \frac{1}{\sqrt{c^{2}-x^{2}}}\right|_{0} ^{v_{2 / 1}}=m_{0} c^{3}\left(\frac{1}{\sqrt{c^{2}-v_{2 / 1}^{2}}}-\frac{1}{c}\right) \\
& =m_{0} c^{2} \frac{1}{\sqrt{1-\left(v_{2 / 1} / c\right)^{2}}}-m_{0} c^{2}
\end{aligned}
$$

This formula is identical to the formula for kinetic energy known from the STR, for the same reasons as in the case of momentum (36).

\subsubsection{The kinetic energy in STR/ $\boldsymbol{F}$ for small velocities}

Formula (41) can be written in the form

$$
\begin{gathered}
E_{2 / 1}^{F}=m_{0} c^{2} \frac{1-\sqrt{1-\left(v_{2 / 1} / c\right)^{2}}}{\sqrt{1-\left(v_{2 / 1} / c\right)^{2}}} \cdot \frac{1+\sqrt{1-\left(v_{2 / 1} / c\right)^{2}}}{1+\sqrt{1-\left(v_{2 / 1} / c\right)^{2}}} \\
E_{2 / 1}^{F}=\frac{m_{0} v_{2 / 1}^{2}}{2} \frac{2}{1-\frac{v_{2 / 1}^{2}}{c^{2}}+\sqrt{1-\frac{v_{2 / 1}^{2}}{c^{2}}}}
\end{gathered}
$$

On this basis, for small values $v_{2 / 1} \ll c$ one receives

$$
v_{2 / 1} \ll c \Rightarrow E_{2 / 1}^{F} \approx \frac{m_{0} v_{2 / 1}^{2}}{2} \frac{2}{1+1}=\frac{m_{0} v_{2 / 1}^{2}}{2}
$$

\subsubsection{The force in STR/F}

Due to the assumption (29) value measurement of the same force by two different observers is identical.

\subsection{STR dynamics with constant momentum change (STR/ $\Delta p)$}

In this section, a model of dynamics of bodies based on the assumption that the change in momentum of the body (parallel to $x$-axis) is the same for an observer from every inertial system will be derived (hence indication $\Delta p$ ).

These dynamics seem particularly interesting, because the conservation law of momentum is a fundamental law. Assumption that the change of body momentum is the same for every observer seems to be a natural extension of this law.

\subsubsection{The relativistic mass in $\mathrm{STR} / \Delta p$}

In the model STR/ $\Delta p$ it is assumed, that

$$
d p_{2 / 1}^{\Delta p}:=d p_{2 / 2}
$$

Having introduced (26) and (27), one obtains

$$
m_{2 / 1}^{\Delta p} d v_{2 / 1}=m_{0} d v_{2 / 2}
$$

On the base (20), one has

$$
m_{2 / 1}^{\Delta p} d v_{2 / 1}=m_{0} \frac{d v_{2 / 1}}{1-\left(v_{2 / 1} / c\right)^{2}}
$$

Hence, a formula for relativistic mass of the body that is located in the system $U_{2}$ and is seen from the system $U_{1}$ is obtained, when assumption (45) is satisfied, as below

$$
m_{2 / 1}^{\Delta p}=m_{0} \frac{1}{1-\left(v_{2 / 1} / c\right)^{2}}
$$

\subsubsection{The momentum in $\mathrm{STR} / \Delta p$}

The body of rest mass $m_{0}$ is associated with the system $U_{2}$. To determine the momentum of the body relative to the system $U_{1}$ a substitution of (48) to (27) is made

$$
\begin{aligned}
d p_{2 / 1}^{\Delta p} & =m_{2 / 1}^{\Delta p} \cdot d v_{2 / 1}=m_{0} \frac{1}{1-\left(v_{2 / 1} / c\right)^{2}} d v_{2 / 1} \\
& =m_{0} c^{2} \frac{1}{c^{2}-v_{2 / 1}^{2}} d v_{2 / 1}
\end{aligned}
$$

The body momentum is a sum of increases in its momentum, when the body is accelerated from the inertial system $U_{1}$ (the body has velocity 0 ) to the inertial system $U_{2}$ (the body has velocity $v_{2 / 1}$ ), i.e.

$$
p_{2 / 1}^{\Delta p}=m_{0} c^{2} \int_{0}^{v_{2 / 1}} \frac{1}{c^{2}-v_{2 / 1}^{2}} d v_{2 / 1}
$$

From the work [2] (formula 52, p. 160) it is possible to read out, that

$$
\int \frac{d x}{a^{2}-x^{2}}=\frac{1}{2 a} \ln \left|\frac{a+x}{a-x}\right|, \quad a \neq 0
$$

After applying the integral (51) to (50) the formula for the body momentum in $U_{2}$ system and measured by the observer from $U_{1}$ system is received in a form of

$$
p_{2 / 1}^{\Delta p}=\left.m_{0} c^{2} \frac{1}{2 c} \ln \left|\frac{c+x}{c-x}\right|\right|_{0} ^{v_{2 / 1}}=\frac{m_{0} c}{2} \ln \left(\frac{c+v_{2 / 1}}{c-v_{2 / 1}}\right)
$$

\subsubsection{The momentum in $S T R / \Delta p$ for small velocities}

Formula (52) can be written in the form

$$
\begin{aligned}
p_{2 / 1}^{\Delta p} & =\frac{m_{0} v_{2 / 1}}{2} \frac{c}{v_{2 / 1}} \ln \left(\frac{c+v_{2 / 1}}{c-v_{2 / 1}}\right) \\
& =\frac{m_{0} v_{2 / 1}}{2} \ln \left(\frac{\left(1+v_{2 / 1} / c\right)^{c / v_{2 / 1}}}{\left(1-v_{2 / 1} / c\right)^{c / v_{2 / 1}}}\right)
\end{aligned}
$$




$$
p_{2 / 1}^{\Delta p}=\frac{m_{0} v_{2 / 1}}{2} \ln \left(\frac{\left(1+\frac{1}{c / v_{2 / 1}}\right)^{c / v_{2 / 1}}}{\left(1-\frac{1}{c / v_{2 / 1}}\right)^{c / v_{2 / 1}}}\right)
$$

On this basis, for small values $v_{2 / 1}<<c$ one receives

$$
\begin{aligned}
v_{2 / 1} \ll c \Rightarrow p_{2 / 1}^{\Delta p} & \approx \frac{m_{0} v_{2 / 1}}{2} \ln \left(\frac{e}{1 / e}\right) \\
& =\frac{m_{0} v_{2 / 1}}{2} \ln \left(e^{2}\right)=m_{0} v_{2 / 1}
\end{aligned}
$$

\subsubsection{The kinetic energy in $\mathrm{STR} / \Delta p$}

A determination of the formula for kinetic energy will be given. The dependence for the relativistic mass (48) is introduced to the formula (28)

$$
\begin{aligned}
d E_{2 / 1}^{\Delta p} & =m_{2 / 1}^{\Delta p} \cdot v_{2 / 1} \cdot d v_{2 / 1}=m_{0} \frac{1}{1-\left(v_{2 / 1} / c\right)^{2}} v_{2 / 1} d v_{2 / 1} \\
& =m_{0} c^{2} \frac{v_{2 / 1}}{c^{2}-v_{2 / 1}^{2}} d v_{2 / 1}
\end{aligned}
$$

The kinetic energy of body is a sum of increases in its kinetic energy, when the body is accelerated from the inertial system $U_{1}$ (the body has velocity 0 ) to the inertial system $U_{2}$ (the body has velocity $v_{2 / 1}$ ), i.e.

$$
E_{2 / 1}^{\Delta p}=m_{0} c^{2} \int_{0}^{v_{2 / 1}} \frac{v_{2 / 1}}{c^{2}-v_{2 / 1}^{2}} d v_{2 / 1}
$$

From the work [2] (formula 56, p. 160) it is possible to read out, that

$$
\int \frac{x}{a^{2}-x^{2}} d x=-\frac{1}{2} \ln \left|a^{2}-x^{2}\right|
$$

After applying the integral (58) to (57) the formula for the kinetic energy of the body in $U_{2}$ system and measured by the observer from $U_{1}$ system in a form of

$$
\begin{gathered}
E_{2 / 1}^{\Delta p}=-\left.m_{0} c^{2} \frac{1}{2} \ln \left|c^{2}-x^{2}\right|\right|_{0} ^{v_{2 / 1}} \\
=-\frac{m_{0} c^{2}}{2} \ln \left(c^{2}-v_{2 / 1}^{2}\right)+\frac{m_{0} c^{2}}{2} \ln \left(c^{2}\right) \\
E_{2 / 1}^{\Delta p}=\frac{m_{0} c^{2}}{2} \ln \frac{c^{2}}{c^{2}-v_{2 / 1}^{2}}=\frac{m_{0} c^{2}}{2} \ln \frac{1}{1-\left(v_{2 / 1} / c\right)^{2}}
\end{gathered}
$$

\subsubsection{The kinetic energy in STR/ $\Delta p$ for small velocities}

Formula (60) can be written in the form

$$
\begin{aligned}
E_{2 / 1}^{\Delta p} & =\frac{m_{0} v_{2 / 1}^{2}}{2} \frac{c^{2}}{v_{2 / 1}^{2}} \ln \frac{1}{1-\left(v_{2 / 1} / c\right)^{2}} \\
& =\frac{m_{0} v_{2 / 1}^{2}}{2} \ln \frac{1}{\left[1-\left(v_{2 / 1} / c\right)^{2}\right]^{\left(c / v_{2 / 1}\right)^{2}}} \\
E_{2 / 1}^{\Delta p} & =\frac{m_{0} v_{2 / 1}^{2}}{2} \ln \frac{1}{\left[1-\frac{1}{\left(c / v_{2 / 1}\right)^{2}}\right]^{\left(c / v_{2 / 1}\right)^{2}}}
\end{aligned}
$$

On this basis, for small values $v_{2 / 1}<<c$ one receives

$$
v_{2 / 1} \ll c \Rightarrow E_{2 / 1}^{\Delta p} \approx \frac{m_{0} v_{2 / 1}^{2}}{2} \ln \frac{1}{1 / e}=\frac{m_{0} v_{2 / 1}^{2}}{2}
$$

\subsubsection{The force in STR/ $\Delta p$}

Body with rest mass $m_{0}$ is related to $U_{2}$ system. It is affected by force that causes acceleration. For the observer from this system, the acceleration force has in accordance with (24) the following value

$$
F_{2 / 2}=m_{0} \frac{d v_{2 / 2}}{d t_{2}}
$$

For the observer from $U_{1}$ system, acceleration force has in accordance with (25) the following value

$$
F_{2 / 1}^{\Delta p}=m_{2 / 1}^{\Delta p} \frac{d v_{2 / 1}}{d t_{1}}
$$

If to divide parties' equation (65) by (64), then on the basis of (20) and (23) one will receive

$$
\frac{F_{2 / 1}^{\Delta p}}{F_{2 / 2}}=\frac{m_{2 / 1}^{\Delta p}}{m_{0}} \cdot \frac{d t_{2}}{d t_{1}} \cdot \frac{d v_{2 / 1}}{d v_{2 / 2}}=\frac{m_{2 / 1}^{\Delta p}}{m_{0}}\left(1-\left(v_{2 / 1} / c\right)^{2}\right)^{3 / 2}
$$

On the basis of (48) a relation between measurements of the same force by two different observers is obtained

$$
F_{2 / 1}^{\Delta p}=\sqrt{1-\left(v_{2 / 1} / c\right)^{2}} \cdot F_{2 / 2}
$$

The highest value of force is measured by the observer from the inertial system in which the body is located.

\subsection{STR dynamics with constant mass (STR/m)}

In this section, a model of dynamics of bodies, based on the assumption that body weight is the same for an observer from each inertial reference system, will be derived (hence indication $m$ ). 


\subsubsection{The relativistic mass in $\mathrm{STR} / \mathrm{m}$}

In the model STR/ $m$ it is assumed, that

$$
m_{2 / 1}^{m}:=m_{0}
$$

Therefore, for the observer from inertial system $U_{1}$, the body mass in $U_{2}$ system is the same as the rest mass.

\subsubsection{The momentum in STR/m}

The body of rest mass $m_{0}$ is associated with the system $U_{2}$. To determine the momentum of the body relative to the system $U_{1}$ a substitution of (68) to (27)

$$
d p_{2 / 1}^{m}=m_{2 / 1}^{m} \cdot d v_{2 / 1}=m_{0} d v_{2 / 1}
$$

The body momentum is a sum of increases in its momentum, when the body is accelerated from the inertial system $U_{1}$ (the body has velocity 0 ) to the inertial system $U_{2}$ (the body has velocity $v_{2 / 1}$ ), i.e.

$$
p_{2 / 1}^{m}=m_{0} \int_{0}^{v_{2 / 1}} d v_{2 / 1}=m_{0} v_{2 / 1}
$$

In this relativistic dynamics the momentum is expressed with the same equation as in classical mechanics.

\subsubsection{The kinetic energy in STR/m}

A determination of the formula for kinetic energy will be given. The dependence for the relativistic mass (68) is introduced to the formula (28)

$$
d E_{2 / 1}^{m}=m_{2 / 1}^{m} \cdot v_{2 / 1} \cdot d v_{2 / 1}=m_{0} v_{2 / 1} d v_{2 / 1}
$$

The kinetic energy of body is a sum of increases in its kinetic energy, when the body is accelerated from the inertial system $U_{1}$ (the body has velocity 0 ) to the inertial system $U_{2}$ (the body has velocity $v_{2 / 1}$ ), i.e.

$$
E_{2 / 1}^{m}=m_{0} \int_{0}^{v_{2 / 1}} v_{2 / 1} d v_{2 / 1}=\frac{m_{0} v_{2 / 1}^{2}}{2}
$$

In this relativistic dynamics the kinetic energy is expressed with the same equation as in classical mechanics.

\subsubsection{The force in STR/m}

Body with rest mass $m_{0}$ is related to $U_{2}$ system. It is affected by force that causes acceleration. For the observer from this system, the acceleration force has in accordance with (24) the following value

$$
F_{2 / 2}=m_{0} \frac{d v_{2 / 2}}{d t_{2}}
$$

For the observer from $U_{1}$ system, acceleration force has in accordance with (25) the following value

$$
F_{2 / 1}^{m}=m_{2 / 1}^{m} \frac{d v_{2 / 1}}{d t_{1}}=m_{0} \frac{d v_{2 / 1}}{d t_{1}}
$$

If to divide parties' equation (74) by (73), then on the basis of (20) and (23) one will receive

$$
\frac{F_{2 / 1}^{m}}{F_{2 / 2}}=\frac{d t_{2}}{d t_{1}} \cdot \frac{d v_{2 / 1}}{d v_{2 / 2}}=\left(1-\left(v_{2 / 1} / c\right)^{2}\right)^{3 / 2}
$$

i.e.

$$
F_{2 / 1}^{m}=\left(1-\left(v_{2 / 1} / c\right)^{2}\right)^{3 / 2} \cdot F_{2 / 2}
$$

The highest value of force is measured by the observer from the inertial system in which the body is located.

\subsubsection{Discussion on the STR/m dynamics}

Obtaining a relativistic dynamics, in which there is no relativistic mass, and equations for kinetic energy and momentum are identical as in classical mechanics can be surprising, because in relativistic mechanics it is believed that the accelerated body can achieve maximum speed $c$. However, this dynamics is formally correct.

If the body velocity $v_{2 / 1}$ reaches $c$ value, then according to (76)

$$
F_{2 / 1}^{m}=\left(1-1^{-}\right)^{3 / 2} \cdot F_{2 / 2} \approx 0
$$

In the inertial system $U_{2}$, in which the body is located, can be affected by acceleration force $F_{2 / 2}$ of any, but finite value. However, from a perspective of the inertial system $U_{1}$, towards which the body has $c$ velocity, the same force is zero. This means that from a perspective of $U_{1}$ system, it is not possible to perform work on the body, which will increase its kinetic energy indefinitely. From the relation (72) it results that the kinetic energy, that a body with mass $m_{0}$ and velocity $c$ has, a value has

$$
E_{\max }^{m}=\frac{m_{0} c^{2}}{2}
$$

\subsection{STR dynamics with constant force to its operation time (STR/F/Dt)}

In this section, a model of dynamics of bodies based on the assumption that the force that accelerates of the body (parallel to $x$-axis) divided by the time of operation of this force is the same for an observer from every inertial system will be derived (hence indication $F / \Delta t$ ). 


\subsubsection{The relativistic mass in $S T R / F / \Delta t$}

In the model STR $/ F / \Delta t$ it is assumed, that

$$
\frac{F_{2 / 1}^{F / \Delta t}}{d t_{1}}:=\frac{F_{2 / 2}}{d t_{2}}
$$

Having introduced (24) and (25), one obtains

$$
m_{2 / 1}^{F / \Delta t} \frac{d v_{2 / 1}}{d t_{1}} \frac{1}{d t_{1}}=m_{0} \frac{d v_{2 / 2}}{d t_{2}} \frac{1}{d t_{2}}
$$

On the base (20) and (23), one has

$$
m_{2 / 1}^{F / \Delta t} \frac{d v_{2 / 1}}{d t_{1}^{2}}=m_{0} \frac{\frac{d v_{2 / 1}}{1-\left(v_{2 / 1} / c\right)^{2}}}{\left(1-\left(v_{2 / 1} / c\right)^{2}\right) d t_{1}^{2}}
$$

Hence, a formula for relativistic mass of the body that is located in the system $U_{2}$ and is seen from the system $U_{1}$ is obtained, when assumption (79) is satisfied, as below

$$
m_{2 / 1}^{F / \Delta t}=m_{0}\left[\frac{1}{1-\left(v_{2 / 1} / c\right)^{2}}\right]^{2}
$$

\subsubsection{The momentum in STR/F/ $\Delta t$}

The body of rest mass $m_{0}$ is associated with the system $U_{2}$. To determine the momentum of the body relative to the system $U_{1}$ a substitution of (82) to (27)

$$
\begin{aligned}
d p_{2 / 1}^{F / \Delta t} & =m_{2 / 1}^{F / \Delta t} \cdot d v_{2 / 1}=m_{0}\left[\frac{1}{1-\left(v_{2 / 1} / c\right)^{2}}\right]^{2} d v_{2 / 1} \\
& =m_{0} c^{4} \frac{1}{\left(c^{2}-v_{2 / 1}^{2}\right)^{2}} d v_{2 / 1}
\end{aligned}
$$

The body momentum is a sum of increases in its momentum, when the body is accelerated from the inertial system $U_{1}$ (the body has velocity 0 ) to the inertial system $U_{2}$ (the body has velocity $v_{2 / 1}$ ), i.e.

$$
p_{2 / 1}^{F / \Delta t}=m_{0} c^{4} \int_{0}^{v_{2 / 1}} \frac{1}{\left(c^{2}-v_{2 / 1}^{2}\right)^{2}} d v_{2 / 1}
$$

From the work [2] (formula 54, p. 160) it is possible to read out, that

$$
\int \frac{d x}{\left(a^{2}-x^{2}\right)^{2}}=\frac{x}{2 a^{2}\left(a^{2}-x^{2}\right)}+\frac{1}{4 a^{3}} \ln \left|\frac{a+x}{a-x}\right|, \quad a \neq 0
$$

After applying the integral (85) to (84) the formula for the body momentum in $U_{2}$ system and measured by the observer from $U_{1}$ system in a form of

$$
\begin{aligned}
p_{2 / 1}^{F / \Delta t} & =\left.m_{0} c^{4}\left[\frac{x}{2 c^{2}\left(c^{2}-x^{2}\right)}+\frac{1}{4 c^{3}} \ln \frac{(c+x)}{(c-x)}\right]\right|_{0} ^{v_{2 / 1}} \\
& =m_{0} c\left[\frac{c v_{2 / 1}}{2\left(c^{2}-v_{2 / 1}^{2}\right)}+\frac{1}{4} \ln \frac{\left(c+v_{2 / 1}\right)}{\left(c-v_{2 / 1}\right)}\right]
\end{aligned}
$$

$$
p_{2 / 1}^{F / \Delta t}=m_{0} v_{2 / 1} \frac{1}{2}\left[\frac{1}{1-\left(v_{2 / 1} / c\right)^{2}}+\ln \left(\frac{c+v_{2 / 1}}{c-v_{2 / 1}}\right)^{\frac{c}{2 v_{2 / 1}}}\right]
$$

\subsubsection{The momentum in STR/F/ $\Delta t$ for small velocities}

Formula (87) can be written in the form

$$
\begin{aligned}
& p_{2 / 1}^{F / \Delta t}= \\
& m_{0} v_{2 / 1}\left[\frac{1}{2\left(1-\left(v_{2 / 1} / c\right)^{2}\right)}+\frac{1}{4} \ln \left(\frac{\left(1+v_{2 / 1} / c\right)^{c / v_{2 / 1}}}{\left(1-v_{2 / 1} / c\right)^{c / v_{2 / 1}}}\right)\right]
\end{aligned}
$$

$$
\begin{aligned}
& p_{2 / 1}^{F / \Delta t}= \\
& m_{0} v_{2 / 1}\left[\frac{1}{2\left(1-\left(v_{2 / 1} / c\right)^{2}\right)}+\frac{1}{4} \ln \left(\frac{\left(1+\frac{1}{c / v_{2 / 1}}\right)^{c / v_{2 / 1}}}{\left(1-\frac{1}{c / v_{2 / 1}}\right)^{c / v_{2 / 1}}}\right)\right]
\end{aligned}
$$

On this basis, for small values $v_{2 / 1}<<c$ one receives

$$
\begin{aligned}
v_{2 / 1} \ll c \Rightarrow p_{2 / 1}^{F / \Delta t} & \approx m_{0} v_{2 / 1}\left[\frac{1}{2}+\frac{1}{4} \ln \left(\frac{e}{1 / e}\right)\right] \\
& =m_{0} v_{2 / 1}\left[\frac{1}{2}+\frac{1}{4} \ln \left(e^{2}\right)\right]=m_{0} v_{2 / 1}
\end{aligned}
$$

\subsubsection{The kinetic energy in STR/F/Dt}

A determination of the formula for kinetic energy will be given. The dependence for the relativistic mass (82) is introduced to the formula (28)

$$
\begin{aligned}
d E_{2 / 1}^{F / \Delta t} & =m_{2 / 1}^{F / \Delta t} \cdot v_{2 / 1} \cdot d v_{2 / 1} \\
& =m_{0}\left[\frac{1}{1-\left(v_{2 / 1} / c\right)^{2}}\right]^{2} v_{2 / 1} d v_{2 / 1} \\
& =m_{0} c^{4} \frac{v_{2 / 1}}{\left(c^{2}-v_{2 / 1}^{2}\right)^{2}} d v_{2 / 1}
\end{aligned}
$$

The kinetic energy of body is a sum of increases in its kinetic energy, when the body is accelerated from the inertial system $U_{1}$ (the body has velocity 0 ) to the inertial system $U_{2}$ (the body has velocity $v_{2 / 1}$ ), i.e.

$$
E_{2 / 1}^{F / \Delta t}=m_{0} c^{4} \int_{0}^{v_{2 / 1}} \frac{v_{2 / 1}}{\left(c^{2}-v_{2 / 1}^{2}\right)^{2}} d v_{2 / 1}
$$


From the work [2] (formula 58, p. 160) it is possible to read out, that

$$
\int \frac{x d x}{\left(a^{2}-x^{2}\right)^{2}}=\frac{1}{2\left(a^{2}-x^{2}\right)}
$$

After applying the integral (93) do (92) the formula for the kinetic energy of the body in $U_{2}$ system and measured by the observer from $U_{1}$ system in a form of

$E_{2 / 1}^{F / \Delta t}=\left.m_{0} c^{4} \frac{1}{2\left(c^{2}-x^{2}\right)}\right|_{0} ^{v_{2 / 1}}=\frac{m_{0} c^{4}}{2} \frac{1}{\left(c^{2}-v_{2 / 1}^{2}\right)}-\frac{m_{0} c^{4}}{2} \frac{1}{c^{2}}$

$E_{2 / 1}^{F / \Delta t}=\frac{m_{0} c^{2}}{2} \frac{1}{1-\left(v_{2 / 1} / c\right)^{2}}-\frac{m_{0} c^{2}}{2}=\frac{m_{0} v_{2 / 1}^{2}}{2} \frac{1}{1-\left(v_{2 / 1} / c\right)^{2}}$

The formula for kinetic energy (95) was derived from the work [3], due to the fact that the author adopted a different assumption than the one on which the dynamics known from the STR was based.

\subsubsection{The kinetic energy in STR/F/ $\Delta t$ for small velocities}

For small velocity $v_{2 / 1} \ll c$ kinetic energy (95) comes down to the kinetic energy from classical mechanics, because

$$
v_{2 / 1} \ll c \Rightarrow E_{2 / 1}^{F / \Delta t} \approx \frac{m_{0} v_{2 / 1}^{2}}{2} \cdot \frac{1}{1}=\frac{m_{0} v_{2 / 1}^{2}}{2}
$$

\subsubsection{The force in STR/F/ $\Delta t$}

Body with rest mass $m_{0}$ is related to $U_{2}$ system. It is affected by force that causes acceleration. For the observer from this system, the acceleration force has in accordance with (24) the following value

$$
F_{2 / 2}=m_{0} \frac{d v_{2 / 2}}{d t_{2}}
$$

For the observer from $U_{1}$ system, acceleration force has in accordance with (25) the following value

$$
F_{2 / 1}^{F / \Delta t}=m_{2 / 1}^{F / \Delta t} \frac{d v_{2 / 1}}{d t_{1}}
$$

If to divide parties' equation (98) by (97), then on the basis of (20) and (23) one will receive

$$
\frac{F_{2 / 1}^{F / \Delta t}}{F_{2 / 2}}=\frac{m_{2 / 1}^{F / \Delta t}}{m_{0}} \cdot \frac{d t_{2}}{d t_{1}} \cdot \frac{d v_{2 / 1}}{d v_{2 / 2}}=\frac{m_{2 / 1}^{F / \Delta t}}{m_{0}}\left(1-\left(v_{2 / 1} / c\right)^{2}\right)^{3 / 2}
$$

On the basis of (82) relation between measurements of the same force by two different observers is obtained

$$
F_{2 / 1}^{F / \Delta t}=\frac{1}{\sqrt{1-\left(v_{2 / 1} / c\right)^{2}}} \cdot F_{2 / 2}
$$

The lowest value of force is measured by the observer from the inertial system in which the body is located.

\subsection{STR dynamics with constant mass to elapse of observer's time (STR/m/ $\Delta t)$}

In this subchapter a model of body dynamics will be derived based on the assumption that the body mass divided by the elapse of time in observer system is the same for the observer from each inertial frame of reference (hence indication $m / \Delta t$ ).

\subsubsection{The relativistic mass in $\mathrm{STR} / \mathrm{m} / \Delta t$}

In the model STR $/ m / \Delta t$ it is assumed, that

$$
\frac{m_{2 / 1}^{m / \Delta t}}{d t_{1}}:=\frac{m_{0}}{d t_{2}}
$$

On the base (23), one obtains

$$
\frac{m_{2 / 1}^{m / \Delta t}}{d t_{1}}=\frac{m_{0}}{\sqrt{1-\left(v_{2 / 1} / c\right)^{2}} \cdot d t_{1}}
$$

Hence, a formula for relativistic mass of the body that is located in the system $U_{2}$ and is seen from the system $U_{1}$ is obtained, when assumption (101) is satisfied, as below

$$
m_{2 / 1}^{m / \Delta t}=m_{0} \frac{1}{\sqrt{1-\left(v_{2 / 1} / c\right)^{2}}}
$$

\subsubsection{The momentum in STR $/ m / \Delta t$}

The body of rest mass $m_{0}$ is associated with the system $U_{2}$. To determine the momentum of the body relative to the system $U_{1}$ a substitution of (103) to (27)

$$
\begin{aligned}
d p_{2 / 1}^{m / \Delta t} & =m_{2 / 1}^{m / \Delta t} \cdot d v_{2 / 1}=m_{0} \frac{1}{\sqrt{1-\left(v_{2 / 1} / c\right)^{2}}} d v_{2 / 1} \\
& =m_{0} c \frac{1}{\sqrt{c^{2}-v_{2 / 1}^{2}}} d v_{2 / 1}
\end{aligned}
$$

The body momentum is a sum of increases in its momentum, when the body is accelerated from the inertial 
system $U_{1}$ (the body has velocity 0 ) to the inertial system $U_{2}$ (the body has velocity $v_{2 / 1}$ ), i.e.

$$
p_{2 / 1}^{m / \Delta t}=m_{0} c^{2} \int_{0}^{v_{2 / 1}} \frac{1}{\sqrt{c^{2}-v_{2 / 1}^{2}}} d v_{2 / 1}
$$

From the work [2] (formula 71, p. 167) it is possible to read out, that

$$
\int \frac{d x}{\sqrt{a^{2}-x^{2}}}=\arcsin \frac{x}{a}, \quad a>0
$$

After applying the integral (106) to (105) the formula for the body momentum in $U_{2}$ system and measured by the observer from $U_{1}$ system in a form of

$$
p_{2 / 1}^{m / \Delta t}=\left.m_{0} c \cdot \arcsin \frac{v_{2 / 1}}{c}\right|_{0} ^{v_{2 / 1}}=m_{0} c \cdot \arcsin \frac{v_{2 / 1}}{c}
$$

\subsubsection{The momentum in $\mathrm{STR} / m / \Delta t$ for small velocities}

Formula (107) can be written in the form

$$
p_{2 / 1}^{m / \Delta t}=m_{0} v_{2 / 1} \frac{\arcsin \frac{v_{2 / 1}}{c}}{\frac{v_{2 / 1}}{c}}
$$

On this basis, for small values $v_{2 / 1}<<c$ one receives

$$
v_{2 / 1} \ll c \Rightarrow p_{2 / 1}^{m / \Delta t} \approx m_{0} v_{2 / 1}
$$

\subsubsection{The kinetic energy in STR $/ m / \Delta t$}

A determination of the formula for kinetic energy will be given. The dependence for the relativistic mass (103) is introduced to the formula (28)

$$
\begin{aligned}
d E_{2 / 1}^{m / \Delta t} & =m_{2 / 1}^{m / \Delta t} \cdot v_{2 / 1} \cdot d v_{2 / 1}=m_{0} \frac{1}{\sqrt{1-\left(v_{2 / 1} / c\right)^{2}}} v_{2 / 1} d v_{2 / 1} \\
& =m_{0} c \frac{v_{2 / 1}}{\sqrt{c^{2}-v_{2 / 1}^{2}}} d v_{2 / 1}
\end{aligned}
$$

The kinetic energy of body is a sum of increases in its kinetic energy, when the body is accelerated from the inertial system $U_{1}$ (the body has velocity 0 ) to the inertial system $U_{2}$ (the body has velocity $v_{2 / 1}$ ), i.e.

$$
E_{2 / 1}^{m / \Delta t}=m_{0} c \int_{0}^{v_{2 / 1}} \frac{v_{2 / 1}}{\sqrt{c^{2}-v_{2 / 1}^{2}}} d v_{2 / 1}
$$

From the work [2] (formula 73, p. 167) it is possible to read out, that

$$
\int \frac{x}{\sqrt{a^{2}-x^{2}}} d x=-\sqrt{a^{2}-x^{2}}
$$

After applying the integral (112) do (111) the formula for the kinetic energy of the body in $U_{2}$ system and measured by the observer from $U_{1}$ system in a form of

$E_{2 / 1}^{m / \Delta t}=-\left.m_{0} c \sqrt{c^{2}-v_{2 / 1}^{2}}\right|_{0} ^{v_{2 / 1}}=-m_{0} c \sqrt{c^{2}-v_{2 / 1}^{2}}+m_{0} c \sqrt{c^{2}}$

$E_{2 / 1}^{m / \Delta t}=m_{0} c^{2}-m_{0} c \sqrt{c^{2}-v_{2 / 1}^{2}}=m_{0} c^{2}\left(1-\sqrt{1-\left(v_{2 / 1} / c\right)^{2}}\right)$

\subsubsection{The kinetic energy in STR/m/ $\Delta t$ for small velocities}

Formula (114) can be written in the form

$$
\begin{aligned}
& E_{2 / 1}^{m / \Delta t}= \\
& \frac{m_{0} v_{2 / 1}^{2}}{2} \cdot \frac{2 c^{2}}{v_{2 / 1}^{2}} \cdot \frac{\left(1-\sqrt{1-\left(v_{2 / 1} / c\right)^{2}}\right)\left(1+\sqrt{1-\left(v_{2 / 1} / c\right)^{2}}\right)}{1+\sqrt{1-\left(v_{2 / 1} / c\right)^{2}}}
\end{aligned}
$$

$$
\begin{aligned}
E_{2 / 1}^{m / \Delta t} & =\frac{m_{0} v_{2 / 1}^{2}}{2} \cdot \frac{2 c^{2}}{v_{2 / 1}^{2}} \cdot \frac{1-\left(1-\left(v_{2 / 1} / c\right)^{2}\right)}{1+\sqrt{1-\left(v_{2 / 1} / c\right)^{2}}} \\
& =\frac{m_{0} v_{2 / 1}^{2}}{2} \frac{2}{1+\sqrt{1-\left(v_{2 / 1} / c\right)^{2}}}
\end{aligned}
$$

On this basis, for small values $v_{2 / 1} \ll c$ one receives

$$
v_{2 / 1} \ll c \Rightarrow E_{2 / 1}^{m / \Delta t} \approx \frac{m_{0} v_{2 / 1}^{2}}{2} \cdot \frac{2}{2}=\frac{m_{0} v_{2 / 1}^{2}}{2}
$$

\subsubsection{The force in STR/m/ $/ \Delta t$}

Body with rest mass $m_{0}$ is related to $U_{2}$ system. It is affected by force that causes acceleration. For the observer from this system, the acceleration force has in accordance with (24) the following value

$$
F_{2 / 2}=m_{0} \frac{d v_{2 / 2}}{d t_{2}}
$$

For the observer from $U_{1}$ system, acceleration force has in accordance with (25) the following value

$$
F_{2 / 1}^{m / \Delta t}=m_{2 / 1}^{m / \Delta t} \frac{d v_{2 / 1}}{d t_{1}}
$$

If to divide parties' equation (119) by (118), then on the basis of (20) and (23) one will receive

$$
\frac{F_{2 / 1}^{m / \Delta t}}{F_{2 / 2}}=\frac{m_{2 / 1}^{m / \Delta t}}{m_{0}} \cdot \frac{d t_{2}}{d t_{1}} \cdot \frac{d v_{2 / 1}}{d v_{2 / 2}}=\frac{m_{2 / 1}^{m / \Delta t}}{m_{0}}\left(1-\left(v_{2 / 1} / c\right)^{2}\right)^{3 / 2}
$$


On the basis of (103) relation between measurements of the same force by two different observers is obtained

$$
F_{2 / 1}^{m / \Delta t}=\left(1-\left(v_{2 / 1} / c\right)^{2}\right) \cdot F_{2 / 2}
$$

The highest value of force is measured by the observer from the inertial system in which the body is located.

\section{The general form of dynamics}

In presented examples, assumptions have been adopted which can be written in forms (30), (46), (68), (80) and (101). On this basis, it can be seen that the assumption for relativistic dynamics is as follows

$$
m_{2 / 1}^{\{a, b\}} \frac{d v_{2 / 1}^{a}}{d t_{1}^{b}}=m_{0} \frac{d v_{2 / 2}^{a}}{d t_{2}^{b}}, \quad a, b \in R
$$

The physical meaning of the formula (122) depends on the value of the parameters $a$ and $b$ to be determined. For example, if $a=b=1$, then this formula takes the form (29), equivalent to the form (30), from the first example.

On the basis of (20) and (23) one receives

$$
\begin{aligned}
m_{2 / 1}^{\{a, b\}} \frac{d v_{2 / 1}^{a}}{d t_{1}^{b}} & =m_{0} \frac{\frac{d v_{2 / 1}^{a}}{\left(1-\left(v_{2 / 1} / c\right)^{2}\right)^{a}}}{\left(1-\left(v_{2 / 1} / c\right)^{2}\right)^{b / 2} \cdot d t_{1}^{b}} \\
& =m_{0}\left[\frac{1}{1-\left(v_{2 / 1} / c\right)^{2}}\right]^{a+b / 2} \frac{d v_{2 / 1}^{a}}{d t_{1}^{b}}
\end{aligned}
$$

Markings are adopted

$$
\{x\} \equiv\{a, b\} \quad \wedge \quad x=a+\frac{b}{2} \in R
$$

Now on the basis of (123) the relativistic inertial mass of body in $U_{2}$ system, seen from $U_{1}$ system, when an assumption is fulfilled (122), is expressed in dynamics $\{x\}$ by the following formula

$$
m_{2 / 1}^{\{x\}}=m_{0}\left[\frac{1}{1-\left(v_{2 / 1} / c\right)^{2}}\right]^{x}
$$

Each such relativistic mass defines a different relativistic dynamics.

According to presented examples, based on formulas (27) and (125), the momentum in dynamicsalign $\{x\}$ is expressed by the following formula

$$
\begin{aligned}
p_{2 / 1}^{\{x\}} & =\int_{0}^{v_{2 / 1}} d p_{2 / 1}^{\{x\}}=\int_{0}^{v_{2 / 1}} m_{2 / 1}^{\{x\}} \cdot d v_{2 / 1} \\
& =m_{0} \int_{0}^{v_{2 / 1}}\left[\frac{1}{1-\left(v_{2 / 1} / c\right)^{2}}\right]^{x} d v_{2 / 1}
\end{aligned}
$$

$$
p_{2 / 1}^{\{x\}}=m_{0} c^{2 x} \int_{0}^{v_{2 / 1}} \frac{1}{\left(c^{2}-v_{2 / 1}^{2}\right)^{x}} d v_{2 / 1}
$$

According to presented examples, based on formulas (28) and (125), the kinetic energy in dynamics $\{x\}$ is expressed by the following formula

$$
\begin{aligned}
E_{2 / 1}^{\{x\}} & =\int_{0}^{v_{2 / 1}} d E_{2 / 1}^{\{x\}}=\int_{0}^{v_{2 / 1}} m_{2 / 1}^{\{x\}} \cdot v_{2 / 1} \cdot d v_{2 / 1} \\
& =m_{0} \int_{0}^{v_{2 / 1}}\left[\frac{1}{1-\left(v_{2 / 1} / c\right)^{2}}\right]^{x} v_{2 / 1} d v_{2 / 1} \\
& E_{2 / 1}^{\{x\}}=m_{0} c^{2 x} \int_{0}^{v_{2 / 1}} \frac{v_{2 / 1}}{\left(c^{2}-v_{2 / 1}^{2}\right)^{x}} d v_{2 / 1}
\end{aligned}
$$

According to presented examples, based on formulas (24), (25) and (20), (23), the relation between forces in dynamics $\{x\}$ is expressed by the following formula

$$
\begin{aligned}
\frac{F_{2 / 1}^{\{x\}}}{F_{2 / 2}} & =\frac{m_{2 / 1}^{\{x\}} \frac{d v_{2 / 1}}{d t_{1}}}{m_{0} \frac{d v_{2 / 2}}{d t_{2}}}=\frac{m_{2 / 1}^{\{x\}} \frac{d v_{2 / 1}}{d t_{1}}}{m_{0} \frac{d v_{2 / 1}}{1-\left(v_{2 / 1} / c\right)^{2}} \cdot \frac{1}{\sqrt{1-\left(v_{2 / 1} / c\right)^{2}} \cdot d t_{1}}} \\
& =\frac{m_{2 / 1}^{\{x\}}}{m_{0}}\left(1-\left(v_{2 / 1} / c\right)^{2}\right)^{3 / 2}
\end{aligned}
$$

On the basis of (125) one receives

$$
\begin{aligned}
\frac{F_{2 / 1}^{\{x\}}}{F_{2 / 2}} & =\left[\frac{1}{1-\left(v_{2 / 1} / c\right)^{2}}\right]^{x}\left(1-\left(v_{2 / 1} / c\right)^{2}\right)^{3 / 2} \\
& =\left[\frac{1}{1-\left(v_{2 / 1} / c\right)^{2}}\right]^{x-\frac{3}{2}}
\end{aligned}
$$

On the basis of (25) and (125) the Newton's second law for dynamics $\{x\}$ is obtained

$$
F_{2 / 1}^{\{x\}}=m_{0}\left[\frac{1}{1-\left(v_{2 / 1} / c\right)^{2}}\right]^{x} a_{2 / 1}
$$

\section{Summary of dynamics}

Summary derived formulas for momentum and kinetic energy:

Dynamics $x=0$

$$
\begin{aligned}
& p_{2 / 1}^{m}=m_{0} v_{2 / 1} \\
& E_{2 / 1}^{m}=\frac{m_{0} v_{2 / 1}^{2}}{2}
\end{aligned}
$$




\section{Dynamics $x=1 / 2$}

$$
\begin{aligned}
p_{2 / 1}^{m / \Delta t}=m_{0} c \cdot \arcsin \frac{v_{2 / 1}}{c}=m_{0} v_{2 / 1} \frac{\arcsin \left(v_{2 / 1} / c\right)}{v_{2 / 1} / c} \\
E_{2 / 1}^{m / \Delta t}=m_{0} c^{2}\left(1-\sqrt{1-\left(v_{2 / 1} / c\right)^{2}}\right) \\
=\frac{m_{0} v_{2 / 1}^{2}}{2} \frac{2}{1+\sqrt{1-\left(v_{2 / 1} / c\right)^{2}}}
\end{aligned}
$$

\section{Dynamics $x=1$}

$$
\begin{gathered}
p_{2 / 1}^{\Delta p}=\frac{m_{0} c}{2} \ln \left(\frac{c+v_{2 / 1}}{c-v_{2 / 1}}\right)=m_{0} v_{2 / 1} \ln \left(\frac{c+v_{2 / 1}}{c-v_{2 / 1}}\right)^{\frac{c}{2 v_{2 / 1}}} \\
E_{2 / 1}^{\Delta p}=\frac{m_{0} c^{2}}{2} \ln \frac{1}{1-\left(v_{2 / 1} / c\right)^{2}} \\
=\frac{m_{0} v_{2 / 1}^{2}}{2} \ln \frac{1}{\left[1-\left(v_{2 / 1} / c\right)^{2}\right]^{\left(c / v_{2 / 1}\right)^{2}}}
\end{gathered}
$$

\section{Dynamics $x=3 / 2$ (recognized STR dynamics)}

$$
\begin{gathered}
p_{2 / 1}^{F}=m_{0} v_{2 / 1} \frac{1}{\sqrt{1-\left(v_{2 / 1} / c\right)^{2}}} \\
E_{2 / 1}^{F}=m_{0} c^{2} \frac{1}{\sqrt{1-\left(v_{2 / 1} / c\right)^{2}}}-m_{0} c^{2} \\
=\frac{m_{0} v_{2 / 1}^{2}}{2} \frac{2}{\sqrt{1-\frac{v_{2 / 1}^{2}}{c^{2}}}\left(1+\sqrt{1-\frac{v_{2 / 1}^{2}}{c^{2}}}\right)}
\end{gathered}
$$

\section{Dynamics $x=2$}

$$
p_{2 / 1}^{F / \Delta t}=m_{0} v_{2 / 1} \frac{1}{2}\left[\frac{1}{1-\left(v_{2 / 1} / c\right)^{2}}+\ln \left(\frac{c+v_{2 / 1}}{c-v_{2 / 1}}\right)^{\frac{c}{2 v_{2 / 1}}}\right]
$$

$$
E_{2 / 1}^{F / \Delta t}=\frac{m_{0} c^{2}}{2} \frac{1}{1-\left(v_{2 / 1} / c\right)^{2}}-\frac{m_{0} c^{2}}{2}=\frac{m_{0} v_{2 / 1}^{2}}{2} \frac{1}{1-\left(v_{2 / 1} / c\right)^{2}}
$$

Figure 4 shows compared momentums from derived relativistic dynamics.

Figure 5 shows compared kinetic energies from derived relativistic dynamics.

Figure 6 shows relation between measurements of the same force from derived relativistic dynamics.

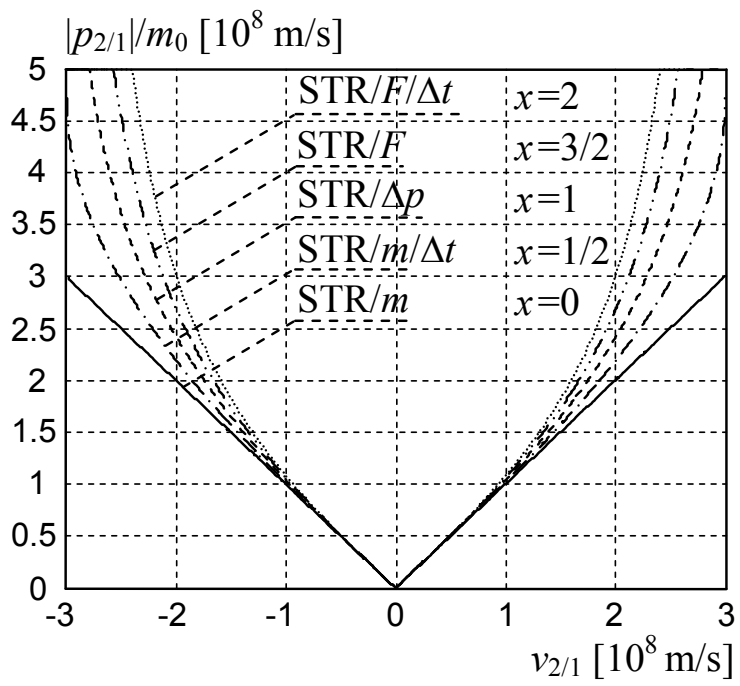

Figure 4: Module of the momentum in dynamics: $\mathrm{STR} / m(x=0), \mathrm{STR} / m / \Delta t(x=1 / 2), \mathrm{STR} / \Delta p(x=1), \mathrm{STR} / F(x=3 / 2)$ and $\mathrm{STR} / F / \Delta t(x=2)$

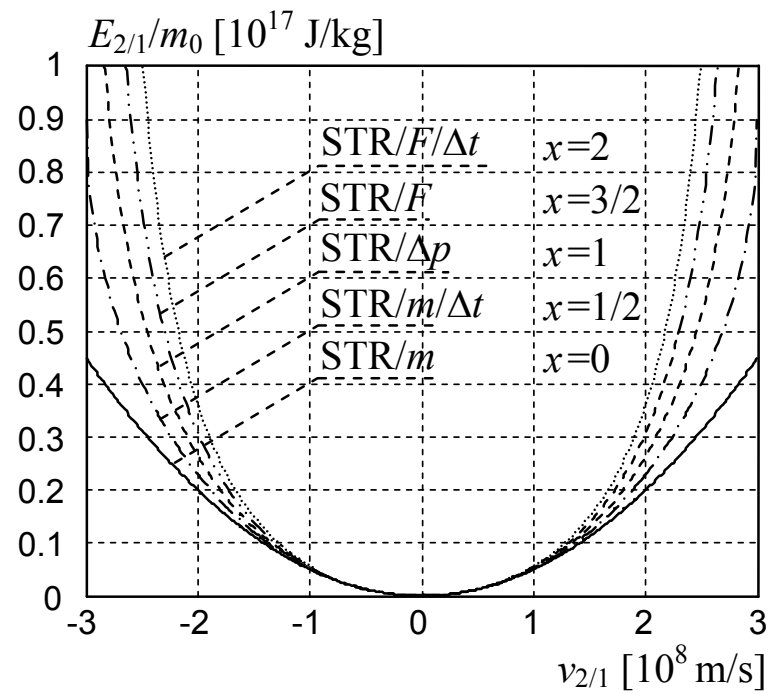

Figure 5: Kinetic energies in dynamics:

$\mathrm{STR} / m(x=0), \mathrm{STR} / m / \Delta t(x=1 / 2), \mathrm{STR} / \Delta p(x=1), \mathrm{STR} / F(x=3 / 2)$ and $\mathrm{STR} / F / \Delta t(x=2)$

\section{Even more general form of dynamics}

Relation (125) to the relativistic mass can be even more generalized. In the general case, it is possible to assume that the relativistic mass is expressed by the following formula

$$
m_{2 / 1}^{\{f\}}=m_{0} \cdot f\left(v_{2 / 1}\right)
$$




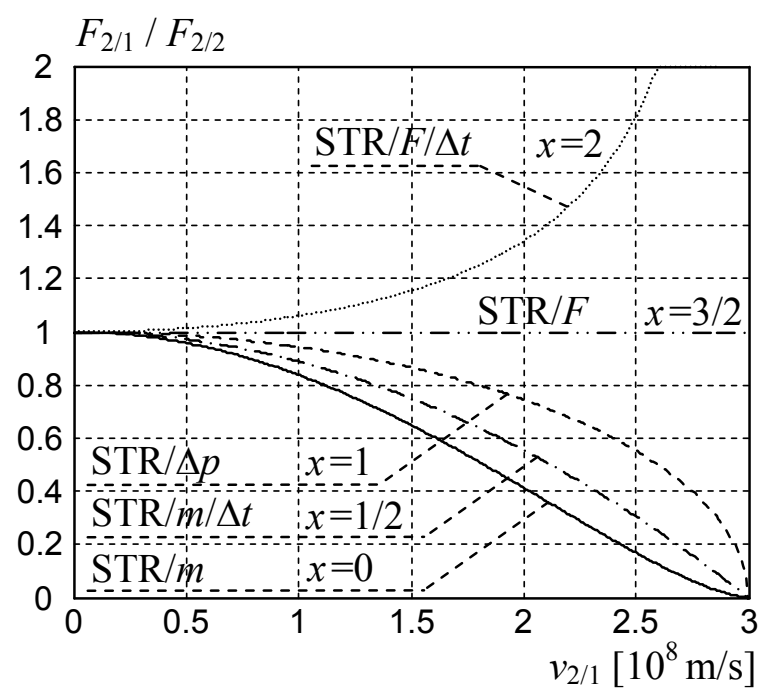

Figure 6: Relation between measurements of the same force by two different observers in dynamics:

$\mathrm{STR} / m(x=0), \mathrm{STR} / m / \Delta t(x=1 / 2), \mathrm{STR} / \Delta p(x=1), \mathrm{STR} / F(x=3 / 2)$ and $\mathrm{STR} / F / \Delta t(x=2)$

Where $f\left(v_{2 / 1}\right)$ is any continuous function with the following properties

$$
\begin{gathered}
f\left(v_{2 / 1}\right) \geq 0 \\
f(0)=1 \\
f\left(v_{2 / 1}\right)=f\left(-v_{2 / 1}\right)
\end{gathered}
$$
the STR.

Each function $f\left(v_{2 / 1}\right)$ defines a different dynamics of

\section{Final conclusions}

This study presents the author's method of deriving dynamics in the Special Theory of Relativity (STR). Five examples of such deriving were shown.

Derivation of dynamics is based on two formulas applicable in the kinematics of STR, i.e. (20) and (23). In order to derive the dynamics of STR, it is necessary to adopt an additional assumption in kinematics, which allows the concept of mass, kinetic energy and momentum to be introduced into the theory.

The dynamics of STR/F $(x=3 / 2)$ is nowadays recognized as the dynamics of the STR. It is based on the assumption that each force parallel to $x$-axis has the same value for the observer from each inertial frame of reference. Formally, however, other dynamics are possible in accordance with the kinematics of the STR. In order to derive them, it is necessary to base on a different assumption.
The currently accepted dynamics of STR has numerous experimental confirmations. However, it is not excluded that more accurate experiments designed specifically for this purpose will show that the optimal model is the dynamics for $x=3 / 2 \pm \Delta x$, where $\Delta x$ is a noticeable correction. A calorimeter can be useful for verification of different dynamics. This device can measure the amount of heat released when stopping particles to high speed. On this basis, it is possible to determine graphs of the kinetic energy of accelerated particles (for example in accelerators of elementary particles) as a function of their velocity, analogous to those presented in Figure 5. On this basis, it is possible to indicate the dynamics in which the kinetic energy of particles is compatible with experiments.

The presented method of dynamism derivation can also be used in other theory of body kinematics. In the monograph [1] this method was used to derive four dynamics in the Special Theory of Ether, which are allowed for kinematics derived in the articles [4, 5]. The presented method is analogous to that used in another area, in the article [6].

\section{References}

[1] Szostek K., Szostek R., Special Theory of Ether, Publishing house AMELIA, Rzeszów, Poland, (www.ste.com.pl), 2015, ISBN 978-83-63359-81-2

[2] Воднев В., Наумович А., Наумович Н., Основные математические формулы. Справочник, 1988, Минск, Издательство «Вышэйшая школа» Государственного комитета БССР, ISBN 5-339-00083-4

[3] Osiak Z., Energy in Special Relativity, Theoretical Physics, Isaac Scient. Publ., 2019, 4 (1), 22-25.

[4] Szostek K., Szostek R., The derivation of the general form of kinematics with the universal reference system, Res. Phys., 2018, 8, 429-437.

[5] Szostek K., Szostek R., Kinematics in Special Theory of Ether, Moscow Univ. Phys. Bull., 2018, 73 (4), 413-421.

[6] Szostek R., An estimation of the geothermal energy sources for generating electricity, Springer, Lecture Notes in Electrical Engineering 324, Analysis and Simulation of Electrical and Computer, 2015, 127-133. 